VoL. 41 (1990) [245-248]

\title{
INEQUALITIES BETWEEN \\ THE INTEGRAL MEANS OF A FUNCTION
}

\author{
D. HAJELA
}

For a measurable function $f$ on a probability space a basic inequality is $\|f\|_{p} \leqslant$ $\|f\|_{q}$, where $1 \leqslant p<q<\infty$ and $\|f\|_{p}$ denotes the $L_{p}$ norm of $f$. The above inequality becomes an equality provided $|f|$ is a constant almost everywhere. We obtain an improvement of the above inequality in all cases that $|f|$ is not a constant almost everywhere.

\section{INTRODUCTION}

Let $\Omega$ be a set with a probability measure $\mu$ on it. For a measurable function $f$ on $\Omega$ and $1 \leqslant p<\infty$ define as usual:

$$
\|f\|_{p}=\left[\int_{\Omega}|f|^{p} d \mu\right]^{1 / p}
$$

and $L_{p}(\Omega)=\left\{f \mid f\right.$ is measurable and $\|f\|_{p}$ is finite $\}$. For $1 \leqslant p<q<\infty$ and a function $f$ in $L_{q}(\Omega)$ a fundamental inequality is $([1])$ :

$$
\|f\|_{p} \leqslant\|f\|_{q}
$$

This inequality is best possible since equality is achieved if and only if $|f|$ is a constant almost everywhere (a.e.). This raises the interesting question of improving upon (1) in the case that $|f|$ is not a constant a.e.. The object of this paper is to give some results doing precisely this. While there has been some work on improving (1) for special values of $p$ and $q$ and also some work on related problems (see Sections 2.14.3 and 2.14.4 in [2] for a number of references), the general problem above does not seem to have been addressed in the literature.

To state the first result define the following: for $a>0$ and $p>1$ let

$$
\delta(a, p)= \begin{cases}{\left[\frac{1}{p}-\frac{1}{p^{2}}\right]\left(a^{p}-1-p \log a\right)} & \text { for } a \geqslant 1 \\ {\left[1-\frac{1}{p}\right](1+a \log a-a)} & \text { for } 0<a \leqslant 1\end{cases}
$$

Received 13 April 1989

Copyright Clearance Centre, Inc. Serial-fee code: 0004-9729/90 \$A2.00+0.00. 
and for $1 \leqslant p<q<\infty$ and a function $f$ in $L_{q}(\Omega)$ let

$$
d(f ; p, q)=\left[1-\int_{\Omega} \delta\left[\frac{|f(x)|^{p}}{\|f\|_{q}^{p}}, \frac{q}{p}\right] d \mu(x)\right]^{1 / p}
$$

We have the following theorem

ThEOREM 3. Let $1 \leqslant p<q<\infty$ and let $f$ be any function in $L_{q}(\Omega)$ such that $|f|$ is not a constant a.e. Then $0<d(f ; p, q)<1$ and $\|f\|_{q} d(f ; p, q) \geqslant\|f\|_{p}$.

This theorem clearly improves upon $(1)$ in all cases where $|f|$ is not a constant a.e.. While it gives a result in terms of the elementary function $\delta(a, p)$, it is possible to get a result which has a simpler form at the expense of getting a weaker result. To state this result, for $1 \leqslant p<q<\infty$ and for a function $f$ in $L_{q}(\Omega)$ define

$$
\Delta(f ; p, q)=\frac{q-p}{q}\left(\|f\|_{q}^{p}-\|f\|_{p}^{p}-\|f\|_{p}^{p} \log \|f\|_{q}^{p}+\int_{\Omega}|f|^{p} \log |f|^{p} d \mu\right) .
$$

THEOREM 4. Let $1 \leqslant p<q<\infty$ and let $f$ be any function in $L_{q}(\Omega)$ such that $|f|$ is not a constant a.e.. Then $\Delta(f ; p, q)>0$ and for $q \geqslant 3^{1 / 3} p,\|f\|_{q}^{p}-\|f\|_{p}^{p} \geqslant$ $\Delta(f ; p, q)$.

The outline of the paper is as follows. In the next section we prove some simple inequalities which are of use in proving the above results. The reader who is so inclined may skip directly to the third section, which contains a proof of the above results, and refer to the inequalities in the next section only as needed.

\section{SOME PRELIMINARY LEMMAS}

In this section we establish some inequalities which will be of use later.

LEMma 1. For $p>1, a>0$ and $\delta(a, p)$ as in (2),

$$
1-\frac{1}{p}+\frac{a^{p}}{p}-a \geqslant \delta(a, p) \text {. }
$$

Proof: Write $\lambda=1 / p$ and $a=e^{x / p}$ for some $-\infty<x<\infty$. Note $0<\lambda<1$. We need to show:

$$
1-\lambda+\lambda e^{x}-e^{\lambda x} \geqslant \begin{cases}\left(\lambda-\lambda^{2}\right)\left(e^{x}-1-x\right) & \text { for } x \geqslant 0 \\ (1-\lambda)\left(1-e^{\lambda x}+\lambda x e^{\lambda x}\right) & \text { for } x \leqslant 0 .\end{cases}
$$

For $x \geqslant 0$, we have that

$$
\begin{aligned}
1-\lambda+\lambda e^{x}-e^{\lambda x} & =1-\lambda+\sum_{k \geqslant 0} \frac{\lambda x^{k}}{k !}-\sum_{k \geqslant 0} \frac{\lambda^{k} x^{k}}{k !} \\
& =\sum_{k \geqslant 2}\left(\lambda-\lambda^{k}\right) \frac{x^{k}}{k !} \geqslant\left(\lambda-\lambda^{2}\right) \sum_{k \geqslant 2} \frac{x^{k}}{k !} \\
& =\left(\lambda-\lambda^{2}\right)\left(e^{x}-1-x\right) .
\end{aligned}
$$


For $x \leqslant 0$, we have using the inequality $1-e^{x} \leqslant-x$ for $x \leqslant 0$ :

$$
\begin{aligned}
1-\lambda+\lambda e^{x}-e^{\lambda x} & =1-\lambda+e^{\lambda x}\left(\lambda e^{(1-\lambda) x}-1\right) \\
& \geqslant 1-\lambda+e^{\lambda x}(\lambda(1+(1-\lambda) x)-1) \\
& =(1-\lambda)\left(1-e^{\lambda x}+\lambda x e^{\lambda x}\right),
\end{aligned}
$$

proving Lemma 1.

LEMMA 2. For $p \geqslant 3^{1 / 3}$ and $a \geqslant 1$,

$$
\left[\frac{1}{p}-\frac{1}{p^{2}}\right]\left(a^{p}-1-p \log a\right) \geqslant\left[1-\frac{1}{p}\right](1+a \log a-a) .
$$

Proof: Write $\lambda=1 / p$ and $a=e^{x / p}$ for some $x \geqslant 0$. Let $f(x)=\left(\lambda-\lambda^{2}\right)\left(e^{x}-1-x\right)$ and $g(x)=(1-\lambda)\left(1+\lambda x e^{\lambda x}-e^{\lambda x}\right)$. We need to show that $f(x)-g(x) \geqslant 0$. Let $h(x)=f(x)-g(x)$. Then $h(0)=0$. Also,

$$
h^{\prime}(x)=\left(\lambda-\lambda^{2}\right)\left(e^{x}-1-\lambda x e^{\lambda x}\right)=\left(\lambda-\lambda^{2}\right) \sum_{k \geqslant 1} \frac{x^{k}}{k !}\left(1-k \lambda^{k}\right) .
$$

Since $(\log x) / x$ is increasing for $1 \leqslant x \leqslant e$ and decreasing for $x>e$, it follows that

$$
\max _{k=1,2, \ldots} \frac{\log k}{k}=\max \left[\frac{\log 2}{2}, \frac{\log 3}{3}\right]=\frac{\log 3}{3}
$$

and so $\log 1 / \lambda \geqslant(\log k) / k$. Thus $1-k \lambda^{k} \geqslant 0$ and so $h^{\prime}(x) \geqslant 0$, which implies $h(x) \geqslant 0$.

\section{INEQUALITIES BETWEEN THE INTEGRAL MEANS OF A FUNCTION}

In this section we give the proofs of the results mentioned in the inroduction which improve upon the basic inequality $\|f\|_{p} \leqslant\|f\|_{q}$ for $1 \leqslant p<q<\infty$.

Proof of Theorem 3: First note that $d(f ; p, q)<1$ for any $f$ such that $|f|$ is not a constant a.e., since for any $b>0$ and $s>1, \delta(b, s)>0$ for $b \neq 1$ and $\delta(1, s)=0$. By Lemma 1 ,

$$
1-\frac{1}{r}+\frac{h(x)^{r}}{r}-h(x) \geqslant \delta(h(x), r),
$$

where $r=q / p, h(x)=g(x) /\|g\|_{r}$ and $g(x)=|f(x)|^{p}$. Note that $\|g\|_{r}=\|f\|_{q}^{p}$ and $\int_{\Omega} h(x)^{r} d \mu(x)=1$. Thus integrating the inequality (5) we get,

$$
1-\int_{\Omega} \frac{|f(x)|^{p}}{\|f\|_{q}^{p}} d \mu(x) \geqslant \int_{\Omega} \delta\left[\frac{|f(x)|^{p}}{\|f\|_{q}^{p}}, \frac{q}{p}\right] d \mu(x)
$$


which completes the proof.

Proof of Theorem 4: To see $\Delta(f ; p, q)>0$ unless $|f|$ is a constant a.e.: Let $r=q / p, g(x)=|f(x)|^{p}$ and $h(x)=g(x) /\|g\|_{r}$. Let $e(a)=1-a+a \log a$ for $a>0$ and note that $e(a)>0$ for $a \neq 1$ and $e(1)=0$. Since $r>1$, it follows that for any $x$ such that $h(x) \neq 1$ :

$$
\left[1-\frac{1}{r}\right](1+h(x) \log h(x)-h(x))>0 .
$$

Integrating the last inequality we get,

$$
\frac{q-p}{q}\left[1+\int_{\Omega} \frac{g(x)}{\|g\|_{r}} \log \frac{g(x)}{\|g\|_{r}} d \mu(x)-\int_{\Omega} \frac{g(x)}{\|g\|_{r}} d \mu(x)\right]>0
$$

unless $|g(x)|$ is a constant a.e.. Substituting $g(x)=|f(x)|^{p}$ shows $\Delta(f ; p, q)>0$ unless $|f|$ is a constant a.e..

Next by Lemmas 1 and 2 , we have for $r \geqslant 3^{1 / 3}$,

$$
1-\frac{1}{r}+\frac{h(x)^{r}}{r}-h(x) \geqslant\left(1-\frac{1}{r}\right)(1+h(x) \log h(x)-h(x)) .
$$

Since $\|g\|_{r}=\|f\|_{q}^{p}$ and $\int_{\Omega} h(x)^{r} d \mu(x)=1$, Theorem 4 follows by integrating the inequality (6) and simplifying.

\section{REFERENCES}

[1] G.H. Hardy, J.E. Littlewood and G. Polya, Inequalities (Cambridge University Press, Cambridge, 1983).

[2] D.S. Mitronovic, Analytic Inequalities (Springer-Verlag, Berlin, Heidelberg, New York, 1970).

Bellcore

445 South Street

Morristown, NJ 07960

United States of America 